FTUAM-02-17

IFT-UAM/CSIC-02-20

November 2, 2018

\title{
Exact Standard Model Compactifications from Intersecting Branes
}

\author{
Christos Kokorelis \\ Dep/to de Física Teórica C-XI and Instituto de Física Teórica C-XVI , \\ Universidad Autónoma de Madrid, Cantoblanco, 28049, Madrid, Spain
}

\begin{abstract}
We construct six stack D6-brane vacua (non-supersymmetric) that have at low energy exactly the standard model (with right handed neutrinos). The construction is based on D6-branes intersecting at angles in $D=4$ type toroidal orientifolds of type I strings. Three $U(1)$ 's become massive through their couplings to RR fields and from the three surviving massless $U(1)$ 's at low energies, one is the standard model hypercharge generator. The two extra massless $U(1)$ 's get broken, as suggested recently (hep-th/0205147), by requiring some intersections to respect $N=1$ supersymmetry thus supporting the appearance of massless charged singlets. Proton and lepton number are gauged symmetries and their anomalies are cancelled through a generalized Green-Schwarz mechanism that gives masses to the corresponding gauge bosons through couplings to RR fields. Thus proton is stable and neutrinos are of Dirac type with small masses as a result of a PQ like-symmetry. The models predict the existence of only two supersymmetric particles, superpartners of $\nu_{R}$ 's.
\end{abstract}




\section{Introduction}

Obtaining chiral string constructions where the low energy particle content may be the observable chiral spectrum of the standard model (SM) and gauge interactions, is one of the important directions of string theory research. In this respect semirealistic models have been pursued along those directions, both in the context of 4D $N=1$ heterotic compactifications and in orientifold constructions [1].

In this work, we will examine standard model compactifications in the context of recent constructions [2, 3, which use intersecting branes and give 4D non-SUSY models. So why we have to resort to non-supersymmetric models in our search for realistic string models ? In $N=1$ heterotic orbifold compactifications (HOC) the semirealistic models derived were supersymmetric (SUSY) and included at low energy the MSSM particle content, together with a variety of exotic matter and/or gauge group factors. However, in order to reconcile the observed discrepancy between the unification of gauge couplings in the MSSM at $10^{16} \mathrm{GeV}$ [4 and the string scale at HOC which is of order $10^{17}-10^{18} \mathrm{GeV}$, it was assumed that the observed difference should be attributed to the presence of the string one loop corrections to the $N=1$ gauge coupling constants [5]. Thus even though it was not possible for a single model to be found which realized at low energy only the MSSM it was assumed that this will become possible after an extensive study of the different compactification vacua was performed. However, the goal of obtaining a particular string compactification with only the MSSM content was not realized. On the other hand in type I compactifications (IC) the string scale is a free parameter. In addition, recent results suggest that it is possible in IC to lower the string scale, by having some compact directions transverse to all stacks of branes, in the TeV region even without SUSY [6]. Thus non-SUSY models with a string scale in the $\mathrm{TeV}$ region provide us with a viable alternative to SUSY models.

For models based on intersecting branes the main picture involves localization of fermions in the intersections between branes [7]. In these constructions the introduction of a quantized NS-NS B field [8] effectively produces semirealistic models with three generations [3]. Under T-duality these backgrounds transform to models with magnetic deformations [9, 10].

Recently, an interesting class of models was found that uses four-stacks of branes and gives exactly the SM content at low energies [11. The models were based on D6-branes intersecting at angles on an orientifolded six-torus compactification [2, 3]. These models have an interesting generalization to classes of models with five-stacks of 
branes [12]. The features of the latter models are quite similar to those that constitute the main part of this work. The models of [11, 12] share some common features as proton and lepton number are gauged symmetries surviving as global symmetries to low energies, small neutrino masses and a remarkable Higgs sector that in cases is of the MSSM. On the contrary, while the four stack model is a non-SUSY model and has a variety of sectors with non-SUSY chiral fields, models that have five-stacks have one additional unusual feature. They have some sectors that preserve $N=1$ SUSY, thus a $N=1$ hypermupliplet remains initially massless t, even though the model is overall a non-SUSY one. The latter feature, as we will see, is maintained in the six-stack classes of models presented in this work.

The models of [11] have been extended to describe the first string non-SUSY model examples, of classes of GUT structured models, which give exactly the SM at low energies [13]. These classes of models 2 are based on the Pati-Salam gauge group $S U(4) \otimes S U(2)_{L} \otimes S U(2)_{R}$ and represent at present, the only consistent GUT models in the context of intersecting branes that are able to produce exactly the SM at low energies. These classes of models maintain essential features of [11, 12] and in particular the fact that proton is stable, as the baryon number is an unbroken gauged symmetry, and small neutrino masses. Also the GUT four-stack classes of models share the unusual features of the classes of the five-stack SM's, four-stack GUT's of [12, 13] respectively, that is even though the models are non-SUSY they do allow for some sectors to preserve $N=1$ SUSY. It is quite interesting to note that, even though it was generally believed that in D6 brane orientifolded six torus models it was not possible to find an apparent explanation for lowering the string scale in the $\mathrm{TeV}$ region , in the classes of GUT models of [13] this issue was solved. In particular, the models predict the existence of light weak doublets with mass of order $v^{2} / M_{s}$, that necessarily needs the string scale to be less than $650 \mathrm{GeV}$. The latter results are particularly encouraging as they represent strong predictions in the context of intersecting D-brane scenarios and are directly testable at present or future accelerators. For additional developments with non-SUSY constructions in the context of intersecting branes, see 114, 15, 16, 17, 18, 19, 20, 21, 22. For a $N=1$ SUSY construction, in the context of intersecting branes, and its phenomenology see [23].

In this work, we will discuss compactifications of intersecting D6 branes, with ex-

\footnotetext{
${ }^{1}$ with the scalar component receiving eventually a mass

${ }^{2}$ They are build on a background of four-stacks of D6 branes, intersecting at angles, on an orientifolded $T^{6}$ background

${ }^{3}$ since there were no torus directions transverse to all branes.
} 
actly the SM at low energies that use six-stacks of D-branes on an orientifolded sixtorus. Thus we practically extending the models of [12] by one more $U(1)$ stack and the models of [11] by two more $U(1)$ stacks. The classes of SM's that we discuss in this work possess some general features:

- Even if the classes of models are non-SUSY overall, they have sectors that preserve $N=1$ supersymmetry. This unusual feature has never appeared in the context of string theory before. It appears that the imposition of SUSY at particular sectors, of the non-SUSY models, creates the necessary singlets that break the two extra $U(1)$ 's symmetries leaving at low energy exactly the SM.

- The models predict unexpectedly the existence of only two SUSY particles, superpartners of right handed neutrinos. This is an extraordinary prediction, because the models are non-SUSY overall. It is those superpartners that are responsible for the breaking of the two additional $U(1)$ symmetries. This is a very strong prediction since, given the fact that the classes of models are non-SUSY, the string scale should be low in order for the hierarchy to be stabilized.

- Baryon number (B) is an unbroken gauged $U(1)$ symmetry, with the corresponding gauge boson to receive a mass and the baryon number to survive as a global symmetry to low energies. Thus proton is stable.

- Lepton number (L) is a gauged $U(1)$ symmetry, thus neutrinos have Dirac masses. Lepton number remains as a global symmetry to low energies. The small masses for the neutrinos will come from the existence of chiral condensates breaking the PQ-like symmetry f $U(1)_{b}$. The gauge bosons corresponding to the gauging of $\mathrm{B}$, L get a mass through their couplings to RR fields that are being involved in a generalized Green-Schwarz mechanism.

The paper is organized as follows. In the next section we describe the rules for constructing the six-stack models for the six-torus orientifolded, with D6 branes intersecting at angles, constructions. We present the representation content and the general solutions to RR tadpole cancellation conditions for the classes of models giving rise exactly to the SM at low energies. In section 3 we describe the cancellation of the mixed $U(1)$ gauge anomalies by a dimensional reduction scheme which is equivalent

\footnotetext{
${ }^{4}$ Beyond the SM gauge symmetries.

${ }^{5}$ The same PS like symmetry was responsible for giving small neutrino masses in the models of [11, 12].
} 
to cancellation of the field theory anomaly by its Green-Schwarz amplitude [11]. This mechanism has been used in the context of toroidal models with branes at angles in [15, 11]. In section 4 we describe the electroweak Higgs sector of the models giving our emphasis on the definition of the geometrical quantities that characterize the geometry of the Higgs sector of the model. In section 5 we describe the remarkable effect of how by imposing the condition that $N=1$ SUSY may be preserved by some sectors, breaks the gauge symmetry to the SM itself. In section 6 , we present a case by case analysis of the possible Higgs fields realized in the models as well describing the neutrino mass generation. Our conclusions together with some comments are presented in section 7 .

\section{Exact Standard model compactifications from In- tersecting branes}

The formalism that we will make use in this work is based on type I strings with D9-branes compactified on a six-dimensional orientifolded torus $T^{6}$, where internal background gauge fluxes on the branes are turned on [2, 3]. By performing a T-duality transformation on the $x^{4}, x^{5}, x^{6}$, directions the D9-branes with fluxes are translated into D6-branes intersecting at angles. Also in this framework we note that the branes are not paralled to the orientifold planes. Under the T-duality the $\Omega$ symmetry, where $\Omega$ is the worldvolume parity is transformed into $\Omega \mathcal{R}$, where $\mathcal{R}$ is the reflection on the T-dualized coordinates,

$$
T(\Omega \mathcal{R}) T^{-1}=\Omega \mathcal{R}
$$

We assume that the $\mathrm{D} 6_{a}$-branes wrap 1-cycles $\left(n_{a}^{i}, m_{a}^{i}\right), i=1,2,3$ along each of the ith- $T^{2}$ torus of the factorized $T^{6}$ torus, namely $T^{6}=T^{2} \times T^{2} \times T^{2}$. Thus we allow the six-torus to wrap factorized 3-cycles, so we can unwrap the 3-cycle into products of three 1-cycles, one for each $T^{2}$. Defining the homology of the 3-cycles as

$$
\left[\Pi_{a}\right]=\prod_{i=1}^{3}\left(n_{a}^{i}\left[a_{i}\right]+m_{a}^{i}\left[b_{i}\right]\right)
$$

defines consequently the 3-cycle of the orientifold images as

$$
\left[\Pi_{a^{\star}}\right]=\prod_{i=1}^{3}\left(n_{a}^{i}\left[a_{i}\right]-m_{a}^{i}\left[b_{i}\right]\right)
$$

We note that in the presence of $\Omega \mathcal{R}$ symmetry, each $\mathrm{D} 6_{a}$-brane 1-cycle, must be accompanied by its $\Omega \mathcal{R}$ orientifold image partner $\left(n_{a}^{i},-m_{a}^{i}\right)$. 
The six-stack SM model structure that we consider in this work is based on the stack structure $U(3) \otimes U(2) \otimes U(1)_{c} \otimes U(1)_{d} \otimes U(1)_{e} \otimes U(1)_{f}$ or $S U(3) \otimes S U(2) \otimes$ $U(1)_{a} \otimes U(1)_{b} \otimes U(1)_{c} \otimes U(1)_{d} \otimes U(1)_{e} \otimes U(1)_{f}$ at the string scale.

In addition, the presence of discrete NS B-flux [8] is assumed. Thus, when the Bflux is present, the tori involved are not orthogonal but tilted. In this way the wrapping numbers become the effective tilted wrapping numbers,

$$
\left(n^{i}, m=\tilde{m}^{i}+n^{i} / 2\right) ; n, \tilde{m} \in Z \text {. }
$$

Thus we allow semi-integer values for the m-wrapping numbers.

The chiral sector is computed from a number of different sectors. As usual in these constructions the chiral fermions get localized in the intersections between branes. The possible sectors are 양

- The $a b+b a$ sector: involves open strings stretching between the D6 $6_{a}$ and D6 $b$ branes. Under the $\Omega \mathcal{R}$ symmetry this sector is mapped to $a^{\star} b^{\star}+b^{\star} a^{\star}$ sector. The number, $I_{a b}$, of chiral fermions in this sector, transform in the bifundamental representation $\left(N_{a}, \bar{N}_{a}\right)$ of $U\left(N_{a}\right) \times U\left(N_{b}\right)$, and reads

$$
I_{a b}=\left[\Pi_{a}\right] \cdot\left[\Pi_{b}\right]=\left(n_{a}^{1} m_{b}^{1}-m_{a}^{1} n_{b}^{1}\right)\left(n_{a}^{2} m_{b}^{2}-m_{a}^{2} n_{b}^{2}\right)\left(n_{a}^{3} m_{b}^{3}-m_{a}^{3} n_{b}^{3}\right)
$$

where $I_{a b}$ is the intersection number of the wrapped cycles. Note that we denote the chirality of the fermions as being associated to the sign of $I_{a b}$ intersection, where $I_{a b}>0$ denotes left handed fermions. Moreover, with negative multiplicity we denote the opposite chirality.

- The $a b^{\star}+b^{\star} a$ sector : It involves chiral fermions transforming into the $\left(N_{a}, N_{b}\right)$ representation with multiplicity given by

$$
I_{a b^{\star}}=\left[\Pi_{a}\right] \cdot\left[\Pi_{b^{\star}}\right]=-\left(n_{a}^{1} m_{b}^{1}+m_{a}^{1} n_{b}^{1}\right)\left(n_{a}^{2} m_{b}^{2}+m_{a}^{2} n_{b}^{2}\right)\left(n_{a}^{3} m_{b}^{3}+m_{a}^{3} n_{b}^{3}\right) .
$$

The $\Omega \mathcal{R}$ symmetry transforms this sector to itself.

- the $a a^{\star}$ sector : under the $\Omega R$ symmetry it transforms to itself. In this sector the invariant intersections will give $8 m_{a}^{1} m_{a}^{2} m_{a}^{3}$ fermions in the antisymmetric representation and the non-invariant intersections that come in pairs provide us with $4 m_{a}^{1} m_{a}^{2} m_{a}^{3}\left(n_{a}^{1} n_{a}^{2} n_{a}^{3}-1\right)$ additional fermions in the symmetric and antisymmetric representation of the $U\left(N_{a}\right)$ gauge group. As it will be explained later, these sectors will be absent from our models.

\footnotetext{
${ }^{6}$ We associate the action of $\Omega \mathcal{R}$ on a sector $a, b$, as being associated to its images $a^{\star}, b^{\star}$, respectively.
} 
Any vacuum derived from the previous intersection constraints is subject in addition to constraints coming from RR tadpole cancellation conditions [2, 3]. That demands cancellation of D6-branes charges \, wrapping on three cycles with homology $\left[\Pi_{a}\right]$ and the O6-plane 7 -form charges wrapping on 3-cycles with homology $\left[\Pi_{O_{6}}\right]$. Note that the $\mathrm{RR}$ tadpole cancellation conditions can be expressed in terms of cancellations of $\mathrm{RR}$ charges in homology as

$$
\sum_{a} N_{a}\left[\Pi_{a}\right]+\sum_{\alpha^{\star}} N_{\alpha^{\star}}\left[\Pi_{\alpha^{\star}}\right]-32\left[\Pi_{O_{6}}\right]=0 .
$$

In explicit form, the RR tadpole conditions read

$$
\begin{aligned}
& \sum_{a} N_{a} n_{a}^{1} n_{a}^{2} n_{a}^{3}=16, \\
& \sum_{a} N_{a} m_{a}^{1} m_{a}^{2} n_{a}^{3}=0, \\
& \sum_{a} N_{a} m_{a}^{1} n_{a}^{2} m_{a}^{3}=0, \\
& \sum_{a} N_{a} n_{a}^{1} m_{a}^{2} m_{a}^{3}=0 .
\end{aligned}
$$

That quarantees absense of non-abelian gauge anomalies.

The complete accommodation of the fermion structure of the six-stack SM model can be seen in table (1). Several comments are in order:

- The models accommodate various known low energy gauged symmetries. The latter can be expressed in terms of the $U(1)$ symmetries $Q_{a}, Q_{b}, Q_{c}, Q_{d}, Q_{e}, Q_{f}$. We find the following identifications

$$
\begin{aligned}
\text { Baryon number } & \rightarrow Q_{a}=3 \mathbf{B}, \\
\text { Lepton number } & \rightarrow \mathbf{L}=Q_{d}+Q_{e}+Q_{f}, \\
3(\mathbf{B}-\mathbf{L}) & \rightarrow Q_{a}-3 Q_{d}-3 Q_{e}-3 Q_{f} .
\end{aligned}
$$

Moreover, $Q_{c}=2 I_{3 R}$, where $I_{3 R}$ being the third component of weak isospin. Also, $3(B-L)$ and $Q_{c}$ are free of triangle anomalies. The $U(1)_{b}$ symmetry plays the role of a Peccei-Quinn symmetry in the sence of having mixed $\mathrm{SU}(3)$ anomalies. This symmetry appears to be a general feature, of the model building based orientifolded six-torus constructions with D6 branes intersecting at angles, in the models based on the four- [1] and five-stack SM's [12].

\footnotetext{
${ }^{7}$ Taken together with their orientifold images $\left(n_{a}^{i},-m_{a}^{i}\right)$ wrapping on three cycles of homology class $\left[\Pi_{\alpha^{\prime}}\right]$.
} 


\begin{tabular}{|c|c|c|c|c|c|c|c|c|c|}
\hline Matter Fields & & Intersection & $Q_{a}$ & $Q_{b}$ & $Q_{c}$ & $Q_{d}$ & $Q_{e}$ & $Q_{f}$ & $\mathrm{Y}$ \\
\hline$Q_{L}$ & $(3,2)$ & $I_{a b}=1$ & 1 & -1 & 0 & 0 & 0 & 0 & $1 / 6$ \\
\hline$q_{L}$ & $2(3,2)$ & $I_{a b^{*}}=2$ & 1 & 1 & 0 & 0 & 0 & 0 & $1 / 6$ \\
\hline$U_{R}$ & $3(\overline{3}, 1)$ & $I_{a c}=-3$ & -1 & 0 & 1 & 0 & 0 & 0 & $-2 / 3$ \\
\hline$D_{R}$ & $3(\overline{3}, 1)$ & $I_{a c^{*}}=-3$ & -1 & 0 & -1 & 0 & 0 & 0 & $1 / 3$ \\
\hline$L^{1}$ & $(1,2)$ & $I_{b d}=-1$ & 0 & -1 & 0 & 1 & 0 & 0 & $-1 / 2$ \\
\hline$L^{2}$ & $(1,2)$ & $I_{b e}=-1$ & 0 & -1 & 0 & 0 & 1 & 0 & $-1 / 2$ \\
\hline$L^{3}$ & $(1,2)$ & $I_{b f}=-1$ & 0 & -1 & 0 & 0 & 0 & 1 & $-1 / 2$ \\
\hline$N_{R}^{1}$ & $(1,1)$ & $I_{c d}=1$ & 0 & 0 & 1 & -1 & 0 & 0 & 0 \\
\hline$E_{R}^{1}$ & $(1,1)$ & $I_{c d^{*}}=-1$ & 0 & 0 & -1 & -1 & 0 & 0 & 1 \\
\hline$N_{R}^{2}$ & $(1,1)$ & $I_{c e}=1$ & 0 & 0 & 1 & 0 & -1 & 0 & 0 \\
\hline$E_{R}^{2}$ & $(1,1)$ & $I_{c e^{*}}=-1$ & 0 & 0 & -1 & 0 & -1 & 0 & 1 \\
\hline$N_{R}^{3}$ & $(1,1)$ & $I_{c f}=1$ & 0 & 0 & 1 & 0 & 0 & -1 & 0 \\
\hline$E_{R}^{3}$ & $(1,1)$ & $I_{c f *}=-1$ & 0 & 0 & -1 & 0 & 0 & -1 & 1 \\
\hline \hline
\end{tabular}

Table 1: Low energy fermionic spectrum of the six stack string scale $S U(3)_{C} \otimes S U(2)_{L} \otimes$ $U(1)_{a} \otimes U(1)_{b} \otimes U(1)_{c} \otimes U(1)_{d} \otimes U(1)_{e} \otimes U(1)_{f}$, type I D6-brane model together with its $U(1)$ charges. Note that at low energies only the SM gauge group $S U(3) \otimes S U(2)_{L} \otimes U(1)_{Y}$ survives.

- The study of Green-Schwarz mechanism will show us that Baryon and Lepton number are unbroken gauged symmetries and the corresponding gauge bosons are massive. It is important to notice that baryon and lepton numbers remain as global symmetries to low energies. Thus proton should be stable. Also Majorana masses for right handed neutrinos are not allowed in the models, that is mass terms for neutrinos should be of Dirac type. In the SM only the diagonal combination

$$
\mathbf{L}_{\text {diag }}=L_{e}+L_{\mu}+L_{\tau}
$$

is an exact symmetry, that means $L_{\text {diag }}$ is preserved in each SM interaction. Thus it appears that the six stack SM's offer a very logical explanation for the existence of the various global symmetries that exist in the SM, in particular the fact the $U(1)_{B-L}$ is an exact global symmetry. 
- The mixed anomalies $A_{i j}$ of the six surplus $U(1)$ 's with the non-abelian gauge groups $S U\left(N_{a}\right)$ of the theory cancel through a generalized GS mechanism [26, 25], involving close string modes couplings to worldsheet gauge fields. Two combinations of the $U(1)$ 's are anomalous and become massive, their orthogonal non-anomalous combinations survive, combining to a single $U(1)$ that remains massless, the latter to be identified with the hypercharge generator.

- The structure of intersection numbers which give the parametric form of tadpole solutions is unique for a certain level of stacks of branes. Another choise of intersection numbers at this level of stacks neither produces the correct hypercharge assignments for the SM chiral particles nor is able to produce a general class of solutions like those presented here or in the four-, five- stack SM's [11, 12] respectively.

- The models make use of the constraint

$$
\Pi_{i=1}^{3} m^{i}=0
$$

The latter constraint is essential to cancel the appearance of exotic representations in the model, appearing from sectors in the form $\alpha \alpha^{\star}$, in antisymmetric and symmetric representations of the $U\left(N_{a}\right)$ group.

- The solutions satisfying simultaneously the intersection constraints and the cancellation of the RR crosscap tadpole constraints are given in parametric form in table (2). These solutions represent the most general solution of the RR tadpoles as they depend on six integer parameters $n_{a}^{2}, n_{d}^{2}, n_{e}^{2}, n_{f}^{2}, n_{b}^{1}, n_{c}^{1}$, the phase parameter $\epsilon= \pm 1$, the NS-background parameter $\beta_{i}=1-b_{i}$, which is associated to the presence of the NS B-field by $b_{i}=0,1 / 2$, and the interpolating parameter $\tilde{\epsilon}= \pm 1$ which gives two different classes of RR tadpole solutions.

The multiparameter tadpole solutions appearing in table (2) represent deformations of the D6-brane branes, of table (1), intersecting at angles, within the same homology class of the factorizable three-cycles. The solutions of table (2) in (2.8) satisfy all tadpole equations but the first. The latter becomes ?

$$
\frac{9 n_{a}^{2}}{\beta^{1}}+2 \frac{n_{b}^{1}}{\beta^{2}}+\frac{n_{d}^{2}}{\beta^{1}}+\frac{n_{e}^{2}}{\beta^{1}}+\frac{n_{f}^{2}}{\beta^{1}}+N_{D} \frac{2}{\beta^{1} \beta^{2}}=16 .
$$

Note that we had added the presence of extra $N_{D}$ branes. Their contribution to the

\footnotetext{
${ }^{8}$ We have added an arbitrary number of $N_{D}$ branes which don't contribute to the rest of the tadpoles and intersection number constraints. Thus in terms of the low energy theory they don't have no effect.

${ }^{9}$ We have set for simplicity $\epsilon=\tilde{\epsilon}=1$.
} 


\begin{tabular}{||c||c|c|c||}
\hline \hline$N_{i}$ & $\left(n_{i}^{1}, m_{i}^{1}\right)$ & $\left(n_{i}^{2}, m_{i}^{2}\right)$ & $\left(n_{i}^{3}, m_{i}^{3}\right)$ \\
\hline \hline$N_{a}=3$ & $\left(1 / \beta^{1}, 0\right)$ & $\left(n_{a}^{2}, \epsilon \beta^{2}\right)$ & $(3, \tilde{\epsilon} / 2)$ \\
\hline$N_{b}=2$ & $\left(n_{b}^{1},-\epsilon \beta^{1}\right)$ & $\left(1 / \beta_{2}, 0\right)$ & $(\tilde{\epsilon}, 1 / 2)$ \\
\hline$N_{c}=1$ & $\left(n_{c}^{1}, \epsilon \beta^{1}\right)$ & $\left(1 / \beta^{2}, 0\right)$ & $(0,1)$ \\
\hline$N_{d}=1$ & $\left(1 / \beta^{1}, 0\right)$ & $\left(n_{d}^{2}, \epsilon \beta^{2}\right)$ & $(1,-\tilde{\epsilon} / 2)$ \\
\hline$N_{e}=1$ & $\left(1 / \beta^{1}, 0\right)$ & $\left(n_{e}^{2}, \epsilon \beta^{2}\right)$ & $(1,-\tilde{\epsilon} / 2)$ \\
\hline$N_{f}=1$ & $\left(1 / \beta^{1}, 0\right)$ & $\left(n_{f}^{2}, \epsilon \beta^{2}\right)$ & $(1,-\tilde{\epsilon} / 2)$ \\
\hline
\end{tabular}

Table 2: Tadpole solutions for six-stacks of D6-branes giving rise to, exactly, the standard model gauge group and observable chiral spectrum, at low energies. The solutions depend on six integer parameters, $n_{a}^{2}, n_{d}^{2}, n_{e}^{2}, n_{f}^{2}, n_{b}^{1}, n_{c}^{1}$, the NS-background $\beta^{i}$ and the phase parameter $\epsilon= \pm 1$ and the extra interpolating parameter $\tilde{\epsilon}= \pm 1$. The $\tilde{\epsilon}$ parameter distinguishes the two different classes of tadpole solutions.

$\mathrm{RR}$ tadpole conditions is best described by placing them in the three-factorizable cycle

$$
N_{D}\left(1 / \beta^{1}, 0\right)\left(1 / \beta^{2}, 0\right)\left(2, m_{D}^{3}\right)
$$

where we have set $m_{D}^{3}=0$. The cancellation of tadpoles is better seen, if we choose a numerical set of wrappings, e.g.

$$
n_{a}^{2}=1, n_{b}^{1}=1, n_{c}^{1} \in Z, n_{d}^{2}=-1, n_{e}=-1, n_{f}^{2}=-1, \beta^{1}=1, \beta^{2}=1 .
$$

Within the above choise, all tadpole conditions but the first are satisfied, the latter is satisfied when we add four $D 6$ branes, e.g. $N_{D}=4$ positioned at $(1,0)(1,0)(2,0)$. Thus the tadpole structure $\square$ becomes

\footnotetext{
${ }^{10}$ Note that the parameter $n_{c}^{1}$ should be defined such that its choise is consistent with a tilted tori,
} 


$$
\begin{array}{cc}
N_{a}=3 & (1,0)(1,1)(3,1 / 2) \\
N_{b}=2 & (1,-1)(1,0)(1,1 / 2) \\
N_{c}=1 & \left(n_{c}^{1}, 1\right)(1,0)(0,1) \\
N_{d}=1 & (1,0)(-1,1)(1,-1 / 2) \\
N_{e}=1 & (1,0)(-1,1)(1,-1 / 2) \\
N_{f}=1 & (1,0)(-1,1)(1,-1 / 2)
\end{array}
$$

Actually, the satisfaction of the tadpole conditions is independent of $n_{c}^{1}$. Thus, when all other parameters are fixed, $n_{c}^{1}$ is a global parameter that can vary according to if the first tori is, or not, tilted. Its precise value will be fixed in terms of the remaining tadpole parameters when we determine the tadpole subclass that is associated with the hypercharge embedding of the standard model.

Note that there are always choises of of wrapping numbers that satisfy the RR tadpole constraints without the need of adding extra paralled branes, e.g. the following choise satisfies all RR tadpoles

$$
\begin{array}{r}
n_{a}^{2}=1, n_{b}^{1}=-1, n_{c}^{1} \in 2 Z+1, n_{d}^{2}=0, \\
n_{e}^{2}=0, n_{f}^{2}=0, \beta^{1}=1 / 2, \beta^{2}=1 .
\end{array}
$$

with cycle wrapping numbers $\boxplus$

$$
\begin{array}{lc}
N_{a}=3 & (2,0)(1,1)(3,1 / 2) \\
N_{b}=2 & (-1,-1 / 2)(1,0)(1,1 / 2) \\
N_{c}=1 & \left(n_{c}^{1}, 1 / 2\right)(1,0)(0,1) \\
N_{d}=1 & (2,0)(0,1)(1,-1 / 2) \\
N_{e}=1 & (2,0)(0,1)(1,-1 / 2) \\
N_{f}=1 & (2,0)(0,1)(1,-1 / 2)
\end{array}
$$

- The hypercharge operator corresponding to the spectrum of table (1), is defined as a linear combination of the $U(1)$ gauge groups, $U(1)_{a}, U(1)_{c}, U(1)_{d}, U(1)_{e}, U(1)_{f}$, as

$$
Y=\frac{1}{6} U(1)_{a}-\frac{1}{2} U(1)_{c}-\frac{1}{2} U(1)_{d}-\frac{1}{2} U(1)_{e}-\frac{1}{2} U(1)_{f}
$$

e.g. $n_{c}^{1}=1$.

${ }^{11}$ Another consistent choise will be $\beta^{1}=1, \beta^{2}=1 / 2, n_{d}^{2}=n_{e}^{2}=n_{f}^{2}=1, n_{a}^{2}=1, n_{b}^{1}=1$. We have set for simplicity $\epsilon=\tilde{\epsilon}=1$. 


\section{$3 \quad \mathrm{U}(1)$ anomaly cancelation}

The general form the mixed anomalies $A_{i j}$ of the six $U(1)$ 's with the non-Abelian gauge groups are given by

$$
A_{i j}=\frac{1}{2}\left(I_{i j}-I_{i j^{\star}}\right) N_{i}
$$

From the mixed anomalies of the $U(1)$ 's with the non-abelian gauge groups $S U(3)_{c}$, $S U(2)_{b}$, we conclude that there are two anomaly free combinations $Q_{c}, Q_{a}-3 Q_{d}-$ $3 Q_{e}-3 Q_{f}$. Also the gravitational anomalies cancel since D6-branes never intersect O6-planes. Gauge anomaly cancellation [26] in the orientifolded type I torus models is quaranteed through a generalized GS mechanism [15] that uses the 10-dimensional RR gauge fields $C_{2}$ and $C_{6}$ and gives at four dimensions the following couplings to gauge fields

$$
\begin{aligned}
& N_{a} m_{a}^{1} m_{a}^{2} m_{a}^{3} \int_{M_{4}} B_{2}^{o} \wedge F_{a} ; n_{b}^{1} n_{b}^{2} n_{b}^{3} \int_{M_{4}} C^{o} \wedge F_{b} \wedge F_{b}, \\
& N_{a} n^{J} n^{K} m^{I} \int_{M_{4}} B_{2}^{I} \wedge F_{a} ; n_{b}^{I} m_{b}^{J} m_{b}^{K} \int_{M_{4}} C^{I} \wedge F_{b} \wedge F_{b},
\end{aligned}
$$

where $C_{2} \equiv B_{2}^{o}$ and $B_{2}^{I} \equiv \int_{\left(T^{2}\right)^{J} \times\left(T^{2}\right)^{K}} C_{6}$ with $I=1,2,3$ and $I \neq J \neq K$. We notice that the four dimensional duals of $B_{2}^{o}, B_{2}^{I}$ are defined as:

$$
C^{o} \equiv \int_{\left(T^{2}\right)^{1} \times\left(T^{2}\right)^{2} \times\left(T^{2}\right)^{3}} C_{6} \quad ; C^{I} \equiv \int_{\left(T^{2}\right)^{I}} C_{2},
$$

where $d C^{o}=-\star d B_{2}^{o}, d C^{I}=-\star d B_{2}^{I}$.

The cancellation of triangle anomalies (3.1) derives from the existence of the string amplitude involved in the GS mechanism [25] in four dimensions [26]. The latter amplitude, where the $U(1)_{a}$ gauge field couples to one of the propagating $B_{2}$ fields, that couples to dual scalars, and couples in turn to two $S U(N)$ gauge bosons, is proportional [11] to

$$
-N_{a} m_{a}^{1} m_{a}^{2} m_{a}^{3} n_{b}^{1} n_{b}^{2} n_{b}^{3}-N_{a} \sum_{I} n_{a}^{I} n_{a}^{J} n_{b}^{K} m_{a}^{I} m_{b}^{J} m_{b}^{K}, I \neq J, K
$$

Taking into account the constraint of (2.11) the RR couplings $B_{2}^{I}$ of (3.3) then appear into the following three terms []:

$$
\begin{array}{r}
B_{2}^{1} \wedge\left(\frac{-2 \epsilon \beta^{1}}{\beta^{2}}\right) F^{b}, \\
B_{2}^{2} \wedge\left(\frac{\epsilon \beta^{2}}{\beta^{1}}\right)\left(9 F^{a}+F^{d}+F^{e}+F^{f}\right), \\
B_{2}^{3} \wedge\left(\frac{3 \tilde{\epsilon} n_{a}^{2}}{2 \beta^{1}} F^{a}+\frac{n_{b}^{1}}{\beta^{2}} F^{b}+\frac{n_{c}^{1}}{\beta^{2}} F^{c}-\frac{\tilde{\epsilon} n_{d}^{2}}{2 \beta^{1}} F^{d}-\frac{\tilde{\epsilon} n_{e}^{2}}{2 \beta^{1}} F^{e}-\frac{\tilde{\epsilon} n_{f}^{2}}{2 \beta^{1}} F^{f}\right) .
\end{array}
$$

\footnotetext{
${ }^{12}$ For simplicity we have set $\tilde{\epsilon}=1$.
} 
Also the couplings of the dual scalars $C^{I}$ of $B_{2}^{I}$ that are required to cancel the mixed anomalies of the six $U(1)$ 's with the non-abelian gauge groups $S U\left(N_{a}\right)$ are given by

$$
\begin{array}{r}
\left.C^{1} \wedge\left[\frac{\epsilon \tilde{\epsilon} \beta^{2}}{2 \beta^{1}}\left(F^{a} \wedge F^{a}\right)-\frac{\epsilon \tilde{\epsilon} \beta^{2}}{\beta^{1}}\left(F^{d} \wedge F^{d}\right)-\frac{\epsilon \tilde{\epsilon} \beta^{2}}{2 \beta^{1}} F^{e} \wedge F^{e}-\frac{\epsilon \tilde{\epsilon} \beta^{2}}{2 \beta^{1}} F^{f} \wedge F^{f}\right)\right] \\
C^{2} \wedge\left[\frac{-\epsilon \beta^{1}}{2 \beta^{2}}\left(F^{b} \wedge F^{b}\right)+\frac{\epsilon \beta^{1}}{\beta^{2}}\left(F^{c} \wedge F^{c}\right)\right], \\
C^{o} \wedge\left[\frac{3 n_{a}^{2}}{\beta^{1}}\left(F^{a} \wedge F^{a}\right)+\frac{\tilde{\epsilon} n_{b}^{1}}{\beta^{2}}\left(F^{b} \wedge F^{b}\right)+\frac{n_{d}^{2}}{\beta^{1}}\left(F^{d} \wedge F^{d}\right)+\right. \\
+\frac{n_{e}^{2}}{\beta^{1}}\left(F^{e} \wedge F^{e}+\frac{n_{f}^{2}}{\beta^{1}}\left(F^{f} \wedge F^{e}\right)\right] .
\end{array}
$$

As in the four stack SM [11], or the five stack SM [12], the RR scalar $B_{2}^{0}$ does not couple to any field $F^{i}$, as we have imposed the condition (2.11) which excludes the appearance of any exotic matter representations in the models.

Note that these representations are not necessary in the SM based stack constructions. However, in the context of building a GUT brane model that eventually has to break to the SM they are welcome, as they become the reason for achieving the breaking to the SM 13.

A closer look at (3.6) reveals that there are two anomalous $U(1)$ 's that become massive through their couplings to the RR fields. They are the model independent fields, $U(1)_{b}$ and the combination $9 U(1)_{a}+U(1)_{d}+U(1)_{e}+U(1)_{f}$, which become massive through their couplings to the $\mathrm{RR} 2$-form fields $B_{2}^{1}, B_{2}^{2}$ respectively. Also there is a model dependent, non-anomalous and massive $U(1)$ field coupled to $B_{2}^{3} \mathrm{RR}$ field. Thus the two non-anomalous free combinations are $U(1)_{c}$ and $U(1)_{a}-3 U(1)_{d}-$ $3 U(1)_{e}-3 U(1)_{f}$. In addition, we note that the mixed anomalies $A_{i j}$ are cancelled by the GS mechanism set by the couplings (3.6, 3.7).

The question we have to ask now, is how we can, from the general class of models, associated with the generic SM's of tables (1) and (2), we can separate the subclass of models associated with just the SM hypercharge assignment at low energies.

The generalized Green-Schwarz mechanism that cancels the non-abelian anomalies of the $U(1)$ 's to the non-abelian gauge fields involves couplings of closed RR string 
modes to the $U(1)$ field strengths ${ }^{13}$ in the form

$$
\sum_{a} f_{a}^{i} B_{a} \wedge \operatorname{tr}\left(F_{i}\right)
$$

Finally, the mixture of couplings in the form

$$
A^{i k}+\sum_{a} f_{a}^{i} g_{a}^{k}=0
$$

cancels all non-abelian $U(1)$ gauge anomalies. That means, as was argued in [11], that if we want to keep some $U(1)$ massless we have to keep it decoupled from some closed string mode couplings that can make it massive, that is

$$
\sum_{a}\left(\frac{1}{6} \tilde{f}_{a}^{\alpha}-\frac{1}{2} \tilde{f}_{a}^{c}-\frac{1}{2} \tilde{f}_{a}^{d}-\frac{1}{2} \tilde{f}_{a}^{e}-\frac{1}{2} \tilde{f}_{a}^{f}\right)=0 .
$$

In conclusion, the combination of the $U(1)$ 's which remains light at low energies is

$3\left(n_{a}^{2}+n_{d}^{2}+n_{3}^{2}+n_{f}^{2}\right) \neq 0, \quad Q^{l}=n_{c}^{1}\left(Q_{a}-3 Q_{d}-3 Q_{e}-3 Q_{f}\right)-\frac{3 \beta^{2} \tilde{\epsilon}\left(n_{a}^{2}+n_{d}^{2}+n_{e}^{2}+n_{f}^{2}\right)}{2 \beta_{1}} Q_{c}$.

The subclass of tadpole solutions of (3.12) having the SM hypercharge assignment at low energies is exactly the one, where the combination (3.12) is proportional to (2.18). That is

$$
n_{c}^{1}=\frac{\tilde{\epsilon} \beta_{2}}{2 \beta_{1}}\left(n_{a}^{2}+n_{d}^{2}+n_{e}^{2}+n_{f}^{2}\right) .
$$

Summarizing, we have found that as long as (3.13) holds, we can identify $Q^{l}$ as the hypercharge generator, which gives at the chiral fermions of table (1) their correct SM hypecharge assignments. Moreover, there are two extra anomaly free $U(1)$ 's beyond the hypercharge combination, which read

$$
\begin{gathered}
Q^{(5)}=\frac{3 \tilde{\epsilon}}{2 \beta_{1}}\left(n_{d}^{2}+n_{e}^{2}+n_{d}^{2}\right)\left(Q_{a}-3 Q_{d}-3 Q_{e}-3 Q_{f}\right)+28 n_{c}^{1} Q_{c} \\
Q^{(6)}=Q_{e}-Q_{f}
\end{gathered}
$$

In the next section, we will break these $U(1)$ symmetries by requiring the intersections where the right handed neutrino is localized, to preserve $N=1$ supersymmetry. A

\footnotetext{
${ }^{13}$ In addition, to the couplings of the Poincare dual scalars $\eta_{a}$ of the fields $B_{a}$,

$$
\sum_{a} g_{a}^{k} \eta_{a} \operatorname{tr}\left(F^{k} \wedge F^{k}\right)
$$
}


comment is in order. The $U(1)$ combinations $Q_{d}-Q_{e}, Q_{d}-Q_{f}$ are anomaly free, that is we could have chosen either of them to be the $Q^{(6)}$ generator. Then the only difference with the present choise (3.14) would be a different constraint on the RR tadple cancellation conditions, once we will later impose $N=1$ on an intersection.

Lets us summarize. Up to this point the gauge group content of the model includes, beyond $S U(3) \otimes S U(2) \otimes U(1)_{Y}$, the additional $U(1)$ symmetries, $Q^{(5)}, Q^{(6)}$ under which some of the chiral SM particles of table (1) gets charged. The extra $U(1)$ symmetries will be broken by imposing some open string sectors to respect some amount of SUSY. In the latter case the immediate effect on obtaining just the SM at low energies will be two additional linear conditions on the $R R$ tadpole solutions of table (2). We note that when $n_{c}^{1}=0$, it is possible to have massless in the low energy spectrum both the $U(1)$ generators, $Q_{c}$, and the B-L generator $(1 / 3)\left(Q_{a}-3 Q_{d}-3 Q_{e}-3 Q_{f}\right)$ as long as $n_{c}^{1}=0, n_{a}^{2}=-n_{d}^{2}-n_{e}^{2}-n_{f}^{2}$.

\section{Higgs mechanism on open string sectors}

The mechanism of electroweak symmetry breaking at the string theory level between intersecting branes is not well understood but it is believed to take place either by using open string tachyons [9, 3, 11, 12, 13] between paralled branes or using brane recombination [20]. As the nature of the latter procedure is topological, it cannot be described using field theoretical methods. In this work, we will follow the former method and we leave the latter method for some future study.

\subsection{The angle structure}

In the previous sections we have detailed the appearance in the R-sector of open string excitations with $I_{a b}$ massless chiral fermions in the D-brane intersections that transform under the bifundamental representations $\left(N_{a}, \bar{N}_{b}\right)$. However, in backgrounds with intersecting branes, besides the actual presence of massless fermions at each intersection, we have evident the presence of an equal number of massive scalars (MS), in the NS-sector, in exactly the same representations as the massless fermions 11. The mass

of the these MS is of order of the string scale. In some cases, it is possible that some of those MS may become tachyonic, triggering a potential that looks like the Higgs potential of the SM, especially when their mass, that depends on the angles between the branes, is such that is decreases the world volume of the 3-cycles involved in the recombination process of joining the two branes into a single one [27]. 
The models that we are describing, are based on orientifolded six-tori on type IIA strings. In those configurations the bulk has $\mathcal{N}=4$ SUSY. Lets us now give some details about the open string sector of the models. In order to describe the open string spectrum we introduce a four dimensional twist [15, 11] vector $v_{\theta}$, whose I-th entry is given by $\vartheta_{i j}$, with $\vartheta_{i j}$ the angle between the branes $i$ and $j$-branes. After GSO projection the states are labeled by a four dimensional twisted vector $r+v_{\theta}$, where $\sum_{I} r^{I}=$ odd and $r_{I} \in \mathbf{Z}, \mathbf{Z}+\frac{\mathbf{1}}{\mathbf{2}}$ for $\mathrm{NS}, \mathrm{R}$ sectors respectively. The Lorentz quantum numbers are denoted by the last entry. The mass operator for the states reads:

$$
\alpha^{\prime} M_{i j}^{2}=\frac{Y^{2}}{4 \pi^{2} \alpha^{\prime}}+N_{b o s}(\vartheta)+\frac{(r+v)^{2}}{2}-\frac{1}{2}+E_{i j}
$$

where $E_{i j}$ the contribution to the mass operator from bosonic oscillators, and $N_{\text {osc }}(\vartheta)$ their number operator, with

$$
E_{i j}=\sum_{I} \frac{1}{2}\left|\vartheta_{I}\right|\left(1-\left|\vartheta_{I}\right|\right),
$$

and $Y$ measures the minimum distance between branes for minimum winding states. If we represent the twisted vector $r+v$, by $\left(\vartheta_{1}, \vartheta_{2}, \vartheta_{3}, 0\right)$, in the NS open string sector, the lowest lying states are given $\square$ by:

$$
\begin{array}{cc}
\text { State } & \text { Mass } \\
\left(-1+\vartheta_{1}, \vartheta_{2}, \vartheta_{3}, 0\right) & \alpha^{\prime} M^{2}=\frac{1}{2}\left(-\vartheta_{1}+\vartheta_{2}+\vartheta_{3}\right) \\
\left(\vartheta_{1},-1+\vartheta_{2}, \vartheta_{3}, 0\right) & \alpha^{\prime} M^{2}=\frac{1}{2}\left(\vartheta_{1}-\vartheta_{2}+\vartheta_{3}\right) \\
\left(\vartheta_{1}, \vartheta_{2},-1+\vartheta_{3}, 0\right) & \alpha^{\prime} M^{2}=\frac{1}{2}\left(\vartheta_{1}+\vartheta_{2}-\vartheta_{3}\right) \\
\left(-1+\vartheta_{1},-1+\vartheta_{2},-1+\vartheta_{3}, 0\right) & \alpha^{\prime} M^{2}=1-\frac{1}{2}\left(\vartheta_{1}+\vartheta_{2}+\vartheta_{3}\right)
\end{array}
$$

Also the angles at the thirteen different intersections can be expressed in terms of the tadpole solutions parameters. Let us define the angles :

$$
\begin{array}{r}
\tilde{\theta}_{1}=\frac{1}{\pi} \tan ^{-1} \frac{\beta^{1} R_{2}^{(1)}}{n_{b}^{1} R_{1}^{(1)}}, \theta_{2}=\frac{1}{\pi} \tan ^{-1} \frac{\beta^{2} R_{2}^{(2)}}{n_{a}^{2} R_{1}^{(2)}}, \tilde{\theta}_{3}=\frac{1}{\pi} \tan ^{-1} \frac{R_{2}^{(3)}}{6 R_{1}^{(3)}}, \\
\theta_{1}=\frac{1}{\pi} \tan ^{-1} \frac{\beta^{1} R_{2}^{(1)}}{n_{c}^{1} R_{1}^{(1)}}, \theta_{2}^{\prime}=\frac{1}{\pi} \tan ^{-1} \frac{\beta^{2} R_{2}^{(2)}}{n_{d}^{2} R_{1}^{(2)}}, \theta_{3}=\frac{1}{\pi} \tan ^{-1} \frac{R_{2}^{(3)}}{2 R_{1}^{(3)}}, \\
\tilde{\theta}_{2}=\frac{1}{\pi} \tan ^{-1} \frac{\beta^{2} R_{2}^{(2)}}{n_{e}^{2} R_{1}^{(2)}}, ; \bar{\theta}_{2}=\frac{1}{\pi} \tan ^{-1} \frac{R_{2}^{(2)}}{n_{f}^{2} R_{1}^{(2)}},
\end{array}
$$

where $R_{1,2}^{(i)}$ are the compactification radii for the three $i=1,2,3$ tori, namely projections of the radii onto the $X_{1,2}^{(i)}$ directions when the NS flux B field, $b^{i}$, is turned on and we have chosen for convenience $\epsilon=\tilde{\epsilon}=1$.

\footnotetext{
14 we assumed $0 \leq \vartheta_{i} \leq 1$.
} 

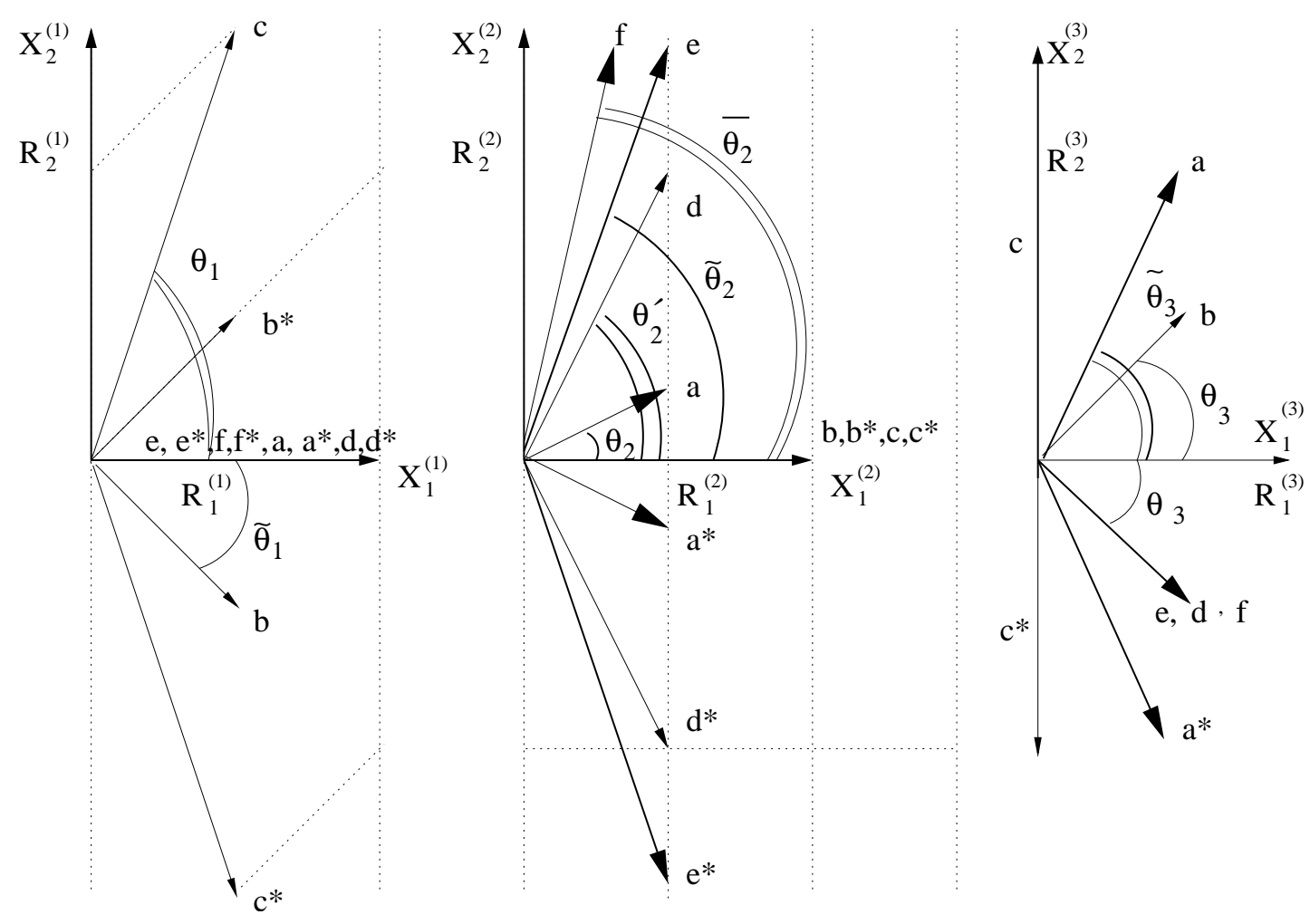

Figure 1: Assignment of angles between D6-branes on the six stack type I model giving rise to the standard model at low energies. The angles between branes are shown on a product of $T^{2} \times T^{2} \times T^{2}$. We have chosen $\beta^{1}=\beta^{2}=1, n_{b}^{1}, n_{c}^{1}, n_{a}^{2}, n_{d}^{2}>0, \epsilon=\tilde{\epsilon}=1$.

At each of the thirteen non-trivial intersections we have the presense of four states $t_{i}, i=1, \cdots, 4$, associated to the states (4.3). Hence we have a total of fifty two different massive scalars, with lowest lying spectrum, in the model 10 .

The following mass relations hold between the different intersections of the model :

$$
\begin{aligned}
m_{a b}^{2}\left(t_{2}\right)+m_{a c}^{2}\left(t_{3}\right) & =m_{c d^{\star}}^{2}\left(t_{2}\right)+m_{c d^{\star}}^{2}\left(t_{3}\right)=m_{c d}^{2}\left(t_{2}\right)+m_{c d}^{2}\left(t_{3}\right) \\
=m_{c e}^{2}\left(t_{1}\right)+m_{c e}^{2}\left(t_{3}\right)= & m_{c e^{\star}}^{2}\left(t_{2}\right)+m_{c e^{\star}}^{2}\left(t_{3}\right)=m_{c f}^{2}\left(t_{2}\right)+m_{c f}^{2}\left(t_{3}\right), \\
m_{a b^{\star}}^{2}\left(t_{2}\right)+m_{a b^{\star}}^{2}\left(t_{3}\right)= & =m_{a b}^{2}\left(t_{2}\right)+m_{a b}^{2}\left(t_{3}\right)=m_{b d}^{2}\left(t_{2}\right)+m_{b d}^{2}\left(t_{3}\right) \\
& =m_{b e}^{2}\left(t_{2}\right)+m_{b e}^{2}\left(t_{3}\right)=m_{b f}^{2}\left(t_{2}\right)+m_{b f}^{2}\left(t_{3}\right), \\
m_{b e}^{2}\left(t_{1}\right)+m_{b e}^{2}\left(t_{2}\right) & =m_{b f}^{2}\left(t_{1}\right)+m_{b f}^{2}\left(t_{2}\right)=m_{b d}^{2}\left(t_{1}\right)+m_{b d}^{2}\left(t_{2}\right)
\end{aligned}
$$

We note that in this work, we will not discuss the stability conditions for absence of tachyonic scalars such that the D-brane configurations dicsussed will be stable as this

\footnotetext{
15 In figure one, we can see the D6 branes angle setup in the present models.
} 
will be discussed elsewhere. Similar conditions have been examined before in [11, 12].

\subsection{Tachyon Higgs mechanism}

In this section, we will study the electroweak Higgs sector of the models. We note that below the string scale the massless spectrum of the model is that of the SM with all particles having the correct hypercharge assignments but with the gauge symmetry being $S U(3) \otimes S U(2) \otimes U(1) \otimes Q^{(5)} \otimes Q^{(6)}$. For the time being we will accept that the, beyond the SM gauge group, $U(1)$ generators break to a scale higher than the scale of electroweak symmetry breaking. The latter issue will be dicsussed in detail in the next section.

In general, tachyonic scalars stretching between two different branes can be used as Higgs scalars as they can become non-tachyonic by varying the distance between paralled branes. In the models presented the complex scalars $h^{ \pm}, H^{ \pm}$get localized between the $b, c$ and between $b, c^{*}$ branes respectively and can be interpreted from the field theory point of view [11] as Higgs fields which are responsible for the breaking the electroweak symmetry. We note that the intersection numbers of the $b, c$ and $b, c^{\star}$ branes across the six-dimensional torus vanish as a result of the fact that the $b, c$ and $b, c^{\star}$ branes are paralled across the second tori. The electroweak Higgs fields, appearing as $H_{i}$ (resp. $\left.h_{i}\right), i=1,2$, in table (3), come from the NS sector, from open strings stretching between the paralled $b, c^{\star}$ (resp. c) branes along the second tori, and from open strings stretching between intersecting branes along the first and third tori.

Initially, the set of Higgs of table (3), are part of the massive spectrum of fields localized in the intersections $b c, b c^{\star}$. However, we note that the Higges $H_{i}, h_{i}$ become massless by varying the distance along the second tori between the $b, c^{\star}, b, c$ branes respectively. Similar set of Higgs fields appear in the four stack [11] and five stack SM's [12 and the Pati-Salam four stack models [13, but obviously with different geometrical data $\square$. We should note that the representations of Higgs fields $H_{i}, h_{i}$ is the maximum allowed by quantization. Their number is model dependent.

For the models presented in this work, the number of complex scalar doublets is equal to the non-zero intersection number product between the $b c, b c^{\star}$ branes in the first and third complex planes. Thus

$$
n_{H^{ \pm}}=I_{b c^{\star}}=\left|\epsilon \tilde{\epsilon} \beta_{1}\left(n_{b}^{1}-n_{c}^{1}\right)\right|, \quad n_{h^{ \pm}}=I_{b c}=\left|\epsilon \tilde{\epsilon} \beta_{1}\left(n_{b}^{1}+n_{c}^{1}\right)\right| .
$$

\footnotetext{
${ }^{16}$ All these models break exactly to the SM at low energies
} 


\begin{tabular}{||c|c||c|c|c||}
\hline \hline Intersection & EW breaking Higgs & $Q_{b}$ & $Q_{c}$ & $\mathrm{Y}$ \\
\hline \hline$b c$ & $h_{1}$ & 1 & -1 & $1 / 2$ \\
\hline$b c$ & $h_{2}$ & -1 & 1 & $-1 / 2$ \\
\hline \hline$b c^{\star}$ & $H_{1}$ & -1 & -1 & $1 / 2$ \\
\hline$b c^{\star}$ & $H_{2}$ & 1 & 1 & $-1 / 2$ \\
\hline \hline
\end{tabular}

Table 3: Higgs fields responsible for electroweak symmetry breaking.

The precise geometrical data for the scalar doublets are

$$
\begin{array}{cc}
\text { State } & \text { Mass }^{2} \\
\left(-1+\vartheta_{1}, 0, \vartheta_{3}, 0\right) & \alpha^{\prime}(\text { Mass })_{Y}^{2}=\frac{Z_{2}}{4 \pi^{2}}+\frac{1}{2}\left(\vartheta_{3}-\vartheta_{1}\right) \\
\left(\vartheta_{1}, 0,-1+\vartheta_{2}, 0\right) & \alpha^{\prime}(\text { Mass })_{X}^{2}=\frac{Z_{3}}{4 \pi^{2}}+\frac{1}{2}\left(\vartheta_{1}-\vartheta_{3}\right)
\end{array}
$$

where $X=\left\{H_{b c^{\star}}^{+}, h_{b c}^{+}\right\}, Y=\left\{H_{b c^{\star}}^{-}, h_{b c}^{-}\right\}$and $Z_{2}$ is the distance ${ }^{2}$ in transverse space along the second torus, $\vartheta_{1}, \vartheta_{3}$ are the (relative) angles between the $b-, c^{\star}$ (for $H^{ \pm}$) (or $b, c$ for $h^{ \pm}$) branes in the first and third complex planes.

As it have been discussed before in [11, 12, 13, 18 the presence of scalar doublets $H^{ \pm}, h^{ \pm}$, can be seen as coming from the field theory mass matrix

$$
\left(\begin{array}{ll}
H_{1}^{*} & H_{2}
\end{array}\right)\left(\mathbf{M}^{2}\right)\left(\begin{array}{c}
H_{1} \\
H_{2}^{*}
\end{array}\right)+\left(\begin{array}{ll}
h_{1}^{*} & h_{2}
\end{array}\right)\left(\mathbf{m}^{2}\right)\left(\begin{array}{c}
h_{1} \\
h_{2}^{*}
\end{array}\right)+h . c .
$$

where

$$
\begin{gathered}
\mathbf{M}^{\mathbf{2}}=M_{s}^{2}\left(\begin{array}{cc}
Z_{2}^{\left(b c^{*}\right)} & \frac{1}{2}\left|\vartheta_{1}^{\left(b c^{*}\right)}-\vartheta_{3}^{\left(b c^{*}\right)}\right| \\
\frac{1}{2}\left|\vartheta_{1}^{\left(b c^{*}\right)}-\vartheta_{3}^{\left(b c^{*}\right)}\right| & Z_{2}^{\left(b c^{*}\right)}
\end{array}\right) \\
\mathbf{m}^{\mathbf{2}}=M_{s}^{2}\left(\begin{array}{cc}
Z_{2}^{(b c)} & \frac{1}{2}\left|\vartheta_{1}^{(b c)}-\vartheta_{3}^{(b c)}\right| \\
\frac{1}{2}\left|\vartheta_{1}^{(b c)}-\vartheta_{3}^{(b c)}\right| & Z_{2}^{(b c)}
\end{array}\right)
\end{gathered}
$$

The fields $H_{i}$ and $h_{i}$ are thus defined as

$$
H^{ \pm}=\frac{1}{2}\left(H_{1}^{*} \pm H_{2}\right) ; h^{ \pm}=\frac{1}{2}\left(h_{1}^{*} \pm h_{2}\right) .
$$

Hence the effective potential which corresponds to the spectrum of Higgs scalars is given by

$$
\begin{array}{r}
V_{\text {Higgs }}=m_{H}^{2}\left(\left|H_{1}\right|^{2}+\left|H_{2}\right|^{2}\right)+m_{h}^{2}\left(\left|h_{1}\right|^{2}+\left|h_{2}\right|^{2}\right) \\
+m_{B}^{2} H_{1} H_{2}+m_{b}^{2} h_{1} h_{2}+\text { h.c. }
\end{array}
$$


where

$$
\begin{gathered}
m_{h}{ }^{2}=\frac{Z_{2}^{(b c)}}{4 \pi^{2} \alpha^{\prime}} \quad ; \quad m_{H}^{2}=\frac{Z_{2}^{\left(b c^{*}\right)}}{4 \pi^{2} \alpha^{\prime}} \\
m_{b}^{2}=\frac{1}{2 \alpha^{\prime}}\left|\vartheta_{1}^{(b c)}-\vartheta_{3}^{(b c)}\right| \quad ; \quad m_{B}^{2}=\frac{1}{2 \alpha^{\prime}}\left|\vartheta_{1}^{\left(b c^{*}\right)}-\vartheta_{3}^{\left(b c^{*}\right)}\right|
\end{gathered}
$$

We note that the $Z_{2}$ is a free parameter, a moduli, and can become very small in relation to the Planck scale. However, the $m_{B}^{2}, m_{b}^{2}$ masses can be expressed in terms of the scalar masses of the particles present at the different intersections $\square$ :

$$
\begin{gathered}
m_{B}^{2}=\frac{1}{2 \alpha^{\prime}}\left|-\tilde{\vartheta}_{1}+\vartheta_{1}-\vartheta_{3}-\frac{1}{2}\right| \quad ; \quad m_{b}^{2}=\frac{1}{2 \alpha^{\prime}}\left|\tilde{\vartheta}_{1}+\vartheta_{1}+\vartheta_{3}-\frac{1}{2}\right| \\
m_{h}^{2}=\frac{1}{2 \alpha^{\prime}}\left(\chi_{b}-\chi_{c}\right)^{2} ; m_{H}^{2}=\frac{1}{2 \alpha^{\prime}}\left(\chi_{b}+\chi_{c}\right)^{2}
\end{gathered}
$$

where $\chi_{b}, \chi_{c}$ the distances from the orientifold plane of the branes b, c. Making use of the scalar mass relations at the intersections of the model we can reexpress the mass relations (4.14), in terms of (4.5). Values of the $m_{B}^{2}, m_{b}^{2}$, in terms of the scalar masses are given in Appendix I.

\section{$5 \quad$ Preserving $N=1$ SUSY at intersections}

Up to this point we have detailed the RR tadpole cancellation solutions for the configuration of the chiral spectrum of table (1) to exist, as well deriving the hypercharge condition (3.13) that quarantees that the hypercharge survives massless to low energies. However, we have not yet derived the gauge interactions corresponding to the observable SM. It remains to be proven that the two additional $U(1)$ 's in (3.14) can be broken. The latter may happen by demanding that some sector preserves $N=1$ SUSY. If this sector supports the presence of a scalar singlet then we can, in principle, break the extra $U(1)$ generator by giving a vev to the singlet. Let us suppose that the sectors $c e$, cf preserve $N=1$ SUSY. In this case, the immediate effect wil be the appearance of $I_{c e}, I_{c f}$ massless scalars at the intersections $c e, c f$ with the same quantum numbers as the massless $I_{c e}, I_{c f}$ fermions. Because $I_{c e}=1, I_{c f}=1$ and the massless fermions localized in the intersections ce, cf respectively are $N_{R}^{2}, N_{R}^{3}$, their massive partners, which will become massless will be their superpartners, the $\tilde{N}_{R}^{2}, \tilde{N}_{R}^{3}$ singlet fields.

Lets us describe the procedure that we will follow in more detail. In order for $N=1$ SUSY to be preserved at some intersection between two branes $\gamma, \delta$ we need to satisfy

\footnotetext{
${ }^{17}$ We have chosen a configuration with $\epsilon=\tilde{\epsilon}=1, n_{b}, n_{c}, n_{d}, n_{e}>0$.
} 
$\pm \vartheta_{1} \pm \vartheta_{2} \pm \vartheta_{3}=0$ for some choise of signs, where $\vartheta_{i}, i=1,2,3$ are the relative angles of the branes $\gamma, \delta$ across the three 2 -tori.

We want the particles localized on the intersection ce to respect some amount of SUSY, in our case $N=1$. That means that the relative angle between branes $c, e$, should obey the SUSY preserving condition

$$
\pm \tilde{\theta}_{1} \pm \bar{\theta}_{2} \pm\left(\frac{\pi}{2}+\theta_{3}\right)=0
$$

So lets us recast the SUSY condition for the ce sector in the form

$$
\tan ^{-1} \frac{\beta_{1} U^{(1)}}{n_{c}^{1}}+\tan ^{-1} \frac{\beta_{2} U^{(2)}}{n_{e}^{2}}-\tan ^{-1}\left(\frac{U^{(3)}}{2}\right)-\frac{\pi}{2}=0,
$$

For the $c f$ sector the SUSY preserving condition

$$
\pm \theta_{1} \pm \theta_{2}^{\prime} \pm\left(\frac{\pi}{2}+\theta_{3}^{\prime}\right)=0
$$

takes the form

$$
\theta_{1}+\theta_{2}^{\prime}-\left(\frac{\pi}{2}+\theta_{3}^{\prime}\right)=0
$$

Conditions (5.2), (5.4) are solved by the choise

$$
n_{e}^{2}=0, \quad n_{f}^{2}=0, ; \frac{\beta_{1} U^{(1)}}{n_{c}^{1}}=\frac{U^{(3)}}{2}, \quad U^{(i)}=\frac{R_{2}^{(i)}}{R_{1}^{(i)}} .
$$

In particular the choise

$$
n_{e}^{2}=0, n_{f}^{2}=0
$$

implies that the second tori is not tilted, e.g. $\beta^{2}=1$.

For a set of Standard Model wrappings to exist we need to consider both the hypercharge (3.13) and the gauge symmetry breaking conditions (5.5). Thus when defining numerically the tadpole solutions of table (2), a consistent set for the observable SM wrapping numbers will be given by

$$
n_{e}^{2}=0, n_{f}^{2}=0, \beta^{2}=1, \beta^{1}=1, n_{b}=-1, n_{d}^{2}=1, n_{a}^{2}=1, n_{c}^{1}=1
$$

or

$$
\begin{array}{cc}
N_{a}=3 & (1,0)(1,1)(3,1 / 2) \\
N_{b}=2 & (-1,-1)(1,0)(1,1 / 2) \\
N_{c}=1 & (1,1)(1,0)(0,1) \\
N_{d}=1 & (1,0)(1,1)(1,-1 / 2) \\
N_{e}=1 & (1,0)(0,1)(1,-1 / 2) \\
N_{f}=1 & (1,0)(0,1)(1,-1 / 2)
\end{array}
$$


The latter choise satisfies all RR tadpole conditions but the first, the latter is satisfied with the addition of three $D 6$ branes located at $(1,0)(1,0)(2,0)$.

After imposing SUSY on sectors $c e, c f$, the $\tilde{N}_{R}^{2}, \tilde{N}_{R}^{3}$ scalar singlets appear. Consequently, as the singlets $\tilde{N}_{R}^{2}, \tilde{N}_{R}^{3}$ get vevs and get charged under $Q^{(5)}, Q^{(6)}$ respectively, break the corresponding $U(1)$ gauge symmetries. Thus the final symmetry leftover is exactly the $S U(3) \otimes S U(2) \otimes U(1)_{Y}$, at low energies. A consequence of the breaking is that this implies the existence of two supersymmetric particles, massive supersymmetric partners of two left handed neutrinos, namely the $\tilde{N}_{R}^{2}, \tilde{N}_{R}^{3}$.

\section{$6 \quad$ Neutrino couplings and masses}

In intersecting brane worlds the size of trilinear Yukawa couplings e.g. between two lepton and a Higgs scalar is controlled by the area of the worldsheet stretching among the three D6-branes crossing at these intersections. That means that the Yukawa couplings in our models are of order [15]

$$
Y_{i j k}=e^{-\tilde{A}_{i j k}}
$$

where $\tilde{A}_{k l m}$ is the worldsheet area, in string units, stretched between the three vertices in the six dimensional compact space. The area of each triangle stretching on each two-dimensional space can be expressed in terms of the lengths of the sides a, b, c of the triangle using the relation 18 , not widely used at present,

$$
A^{(2)}=\sqrt{s(s-a)(s-b)(s-c)}
$$

where $s=(a+b+c) / 2$.

As we have said there are four different electroweak Higgs present in the models $H_{1}, H_{2}, h_{1}, h_{2}$. Thus the full Yukawa interactions for the chiral spectrum of the SM's fields, allowed by all the symmetries, read

$$
\begin{array}{r}
Y_{j}^{U} Q_{L} U_{R}^{j} h_{1}+Y_{j}^{D} Q_{L} D_{R}^{j} H_{2}+ \\
Y_{i j}^{u} q_{L}^{i} U_{R}^{j} H_{1}+Y_{i j}^{d} q_{L}^{i} D_{R}^{j} h_{2}+ \\
\sum_{m=1}^{3} Y_{i j}^{N}\left(L^{m}\right)^{i}\left(N_{R}^{m}\right)^{j} h_{1}+\sum_{m=1}^{3} Y_{i j}^{E}\left(L^{m}\right)^{i}\left(E_{R}^{m}\right)^{j} H_{2}+\text { h.c. }
\end{array}
$$

where $i=1,2, j=1,2,3$.

\footnotetext{
${ }^{18}$ Found by 29]
} 


\begin{tabular}{||c|c|c|c|c|c|c||}
\hline \hline Higgs fields & $\beta_{1}$ & $\beta_{2}$ & $n_{a}^{2}$ & $n_{b}^{1}$ & $n_{c}^{1}$ & $N_{D}$ \\
\hline$n_{H}=1, n_{h}=0$ & $1 / 2$ & 1 & $-1-n_{d}^{2}$ & 1 & -1 & $8+4 n_{d}^{2}$ \\
\hline$n_{H}=1, n_{h}=0$ & $1 / 2$ & 1 & $1-n_{d}^{2}$ & -1 & 1 & $4 n_{d}^{2}$ \\
\hline \hline$n_{H}=0, n_{h}=1$ & $1 / 2$ & 1 & $1-n_{d}^{2}$ & 1 & -1 & $-1-4 n_{d}^{2}$ \\
\hline$n_{h}=0, n_{h}=1$ & $1 / 2$ & 1 & $-1-n_{d}^{2}$ & -1 & -1 & $9+4 n_{d}^{2}$ \\
\hline \hline$n_{H}=1, n_{h}=1$ & 1 & 1 & $2-n_{d}^{2}$ & 0 & 1 & $-1-4 n_{d}^{2}$ \\
\hline$n_{H}=1, n_{h}=1$ & 1 & 1 & $-2-n_{d}^{2}$ & 0 & -1 & $17+4 n_{d}^{2}$ \\
\hline$n_{H}=1, n_{h}=1$ & 1 & 1 & $-n_{d}^{2}$ & 1 & 0 & $7+4 n_{d}^{2}$ \\
\hline$n_{H}=1, n_{h}=1$ & 1 & 1 & $-n_{d}^{2}$ & -1 & 0 & $9+4 n_{d}^{2}$ \\
\hline \hline
\end{tabular}

Table 4: Families of models with minimal Higgs structure. They depend on a single integer, $n_{d}$. The surplus gauge symmetry breaking condition (5.6) has been taken into account.

The scalar doublets in the model present, that are interpreted in terms of the low energy theory as Higgs doublets were given in (4.11). As the number of Higgs present in the models depends on the parameters of the tadpole solutions, the most interesting cases involve the following two possibilities: The 'minimal Higgs presence' case and the 'next to minimal Higgs presence' case, $n_{H}=1, n_{h}=1$.

\section{- Minimal Higgs presence - Higgs system of MSSM}

In this case, we have either $n_{H}=1, n_{h}=0$ or $n_{H}=0, n_{h}=1$. In those cases we can see, see table (4), that there are two families of models left that depend on a single integer, e.g. $n_{d}^{2}$. The solutions in this case are shown in the four top rows of table (4). We also list the number of necessary $N_{D}$ branes required to cancel the first tadpole condition. We have taken into account the conditions (3.13), (5.6) necessary to obtain the observable SM at low energies. The geometrical data for the Higgs system e.g. we choose $n_{H}=1, n_{h}=0$ read :

$$
m_{H}^{2}=\frac{\left(\chi_{b}+\chi_{c}\right)^{2}}{\alpha^{\prime}} ;, \quad m_{B}^{2}=\frac{1}{2 \alpha^{\prime}}\left|\vartheta_{1}-\tilde{\vartheta}_{1}-\vartheta_{3}-\frac{1}{2}\right|
$$

A general feature of the SM models with four [11] of five stacks [13 is the realization of the two Higgs system like in the MSSM. In this minimal case, only the $H_{1}, H_{2}$ Higgs fields appear. For the quark sector the analysis is identical to 
the one appearing in [11, 12]. From (6.3) we conclude that all charged lepton get a tree level mass. Also two u-quarks and one d-quark receive a mass, namely the t, c, b quarks, with masses of order of GeV. The rest of the quarks, don't receive a mass at tree level, as effective couplings in the form $Q_{L} U_{R} H_{1}, q_{L} D_{R} H_{2}$ could violate the $U(1)_{b}$ PQ-like global symmetry. However, this symmetry will be broken by strong interaction effects, effectively giving masses to $\mathrm{u}, \mathrm{d}$, s quarks that have masses less than the QCD scale.

As far as the neutrino masses are concerned, the models admits the following dimension six operators

$$
L^{1} N_{R}^{1}\left(Q_{L} U_{R}\right)^{*}, L^{2} N_{R}^{2}\left(Q_{R} U_{R}\right)^{*}, L^{3} N_{R}^{3}\left(Q_{L} U_{R}\right)^{*}
$$

For values of the u-quark chiral condensate taken from lattice calculations [28], with $<u_{L} u_{R}>\approx(240 \mathrm{MeV})^{3}$, and values of the string scale between $1-10 \mathrm{TeV}$, one can get neutrino values between $0.1-10 \mathrm{eV}$ in consistency with neutrino oscillation experiments.

\section{- Next to minimal Higgs presence}

The next to minimal set of Higges is obtained when $n_{H}=1, n_{h}=1$. In this case, quarks and leptons get their mass from the start. From table (4) we can see that there are two families of models that depend on a single integer $n_{d}^{2}$, we have imposed the conditions (3.13) and (5.6). The geometrical data for this Higgs system read :

$$
\begin{array}{r}
m_{H}^{2}=\frac{\left(\chi_{b}+\chi_{c}\right)^{2}}{\alpha^{\prime}}, \quad m_{h}^{2}=\frac{\left(\chi_{b}-\chi_{c}\right)^{2}}{\alpha^{\prime}} \\
m_{B}^{2}=\frac{1}{2 \alpha^{\prime}}\left|\vartheta_{1}-\vartheta_{3}\right|, m_{b}^{2}=\frac{1}{2 \alpha^{\prime}}\left|\vartheta_{1}+\vartheta_{3}\right|
\end{array}
$$

A hierachy of lepton masses in this case would arise from a hierarchy of the Yukawa couplings and the vevs of Higgs masses involved.

\section{Conclusions and future directions}

In this paper, we have discuss, the construction and the phenomenological properties of a new vacuum of type IIA theory compactified on a orientifolded T6 torus with D6 branes intersecting an angles. This particular construction gives us classes of models that have at low energies exactly the standard model. We note that until recently, 
obtaining the standard model at low energies from a particular string construction was an impossible task.

The models have some important properties including a stable proton, small neutrino masses. Moreover, even if the models have a non-supersymmetric spectrum they predict the existence of exactly two supersymmetric particles, superpartners of two generations right handed neutrinos. This phenomenon, namely the existence of supersymmetric particles in a non-SUSY model is a completely new phenomenon and has appeared before only in the five stack models we discussed in [13]. We note that the existence of SUSY particles, does not appear in the four stack standard models of [11] but it is a feature of its higher stack generalizations ${ }^{\text {ID }}$. The reason that supersymmetry make its appearance is because of the need to create extra singlets into the theory that would break the extra $U(1)$ generators present at low energies.

We also note that it is not possible in the present context 20 of stacks of D6 branes based on an $S U(3) \otimes S U(2) \otimes U(1)^{n}$ content \&, to lower the string scale with any known procedure. This should be contrasted with the classes of models discussed in [13, where we have stacks of D6 branes, in the same compact background, but with stacks of D6 branes based on a GUT like group like $S U(4)_{C} \otimes S U(2)_{L} \otimes S U(2)_{R} \otimes U(1)^{4}$. In the latter case the string scale can be shown to be very low and constrained to be less than $650 \mathrm{GeV}$. The models presented, as well all the models derived from the same six torus backgrounds, have non-vanishing NSNS tadpoles. Thus the question of the full stability ${ }^{2}$ of the models is an open question. It will be interesting to examine numerically the question of full stability of our configurations along the lines of [24, 30, 31]. We remind that the background could be cured in principle be redefining it [32, 33] or by modifying the models such that the NSNS tadpoles may be absent. We hope to return to the last issue in a future work. Moreover, it will be interesting to examine the consequences for the electroweak breaking for the present models and for the 5-stack SM's [12] using brane recombination [19]. We leave this task for a future work.

Recently, it has been pointed out [22 that it is possible, by considering intersecting branes with compactifications of IIA theory on Calabi-Yau 3-folds, to rederive the chiral context of the 4-stack SM configurations of [1]. It will be interesting to examine how

\footnotetext{
${ }^{19}$ See also 12 .

${ }^{20}$ This has been confirmed in the cases of $n=5$ [12] and $n=6$ in the present paper.

${ }^{21}$ moving in a orientifolded six torus background of IIA theory

${ }^{22}$ For some other works related to stability questions on intersecting brane backgrounds see also [11, 12, 24, 31, 30].
} 
the classes of 6-stack chiral configurations examined in the present work and those of 5-stack SM's [12] can be realized explicitly in the Calabi-Yau case.

\section{Acknowledgements}

I am grateful to L. Ibáñez, R. N. Mohapatra, S. Sint and A. Uranga for usuful discussions.

\section{Appendix A}

In this appendix, we list the values of the Higgs mass parameters, of section (4). Their values can be expressed in terms of the scalar masses at the different intersections.

$$
\begin{array}{r}
m_{B}^{2}=\mid-\frac{1}{2}\left(m_{q_{L}}^{2}\left(t_{2}\right)+m_{q_{L}}^{2}\left(t_{3}\right)\right)+\frac{1}{2}\left(m_{U_{R}}^{2}\left(t_{2}\right)+m_{U_{R}}^{2}\left(t_{3}\right)\right)- \\
\frac{1}{4}\left(m_{L^{2}}^{2}\left(t_{1}\right)+m_{L^{2}}^{2}\left(t_{2}\right)\right)-\frac{1}{2} \mid= \\
\mid-\frac{1}{2}\left(m_{Q_{L}}^{2}\left(t_{2}\right)+m_{Q_{L}}^{2}\left(t_{3}\right)\right)+\frac{1}{2}\left(m_{N_{R}^{2}}^{2}\left(t_{1}\right)+m_{N_{R}^{2}}^{2}\left(t_{3}\right)\right)- \\
\frac{1}{4}\left(m_{L^{3}}^{2}\left(t_{1}\right)+m_{L^{3}}^{2}\left(t_{2}\right)\right)-\frac{1}{2} \mid= \\
\mid-\frac{1}{2}\left(m_{L^{1}}^{2}\left(t_{2}\right)+m_{L^{1}}^{2}\left(t_{3}\right)\right)+\frac{1}{2}\left(m_{N_{R}^{3}}^{2}\left(t_{2}\right)+m_{N_{R}^{3}}^{2}\left(t_{3}\right)\right)- \\
\frac{1}{4}\left(m_{L^{1}}^{2}\left(t_{1}\right)+m_{L^{1}}^{2}\left(t_{2}\right)\right)-\frac{1}{2} \mid= \\
\mid-\frac{1}{2}\left(m_{L^{3}}^{2}\left(t_{2}\right)+m_{L^{3}}^{2}\left(t_{3}\right)\right)+\frac{1}{2}\left(m_{N_{R}^{3}}^{2}\left(t_{2}\right)+m_{N_{R}^{3}}^{2}\left(t_{3}\right)\right)- \\
\frac{1}{4}\left(m_{L^{3}}^{2}\left(t_{1}\right)+m_{L^{3}}^{2}\left(t_{2}\right)\right)-\frac{1}{2} \mid= \\
\frac{1}{4}\left(m_{L^{1}}^{2}\left(t_{1}\right)+m_{L^{1}}^{2}\left(t_{2}\right)\right)-\frac{1}{2} \mid= \\
m_{b}^{2}=\mid \begin{array}{r}
\left.\frac{1}{2}\left(m_{q_{L}}^{2}\left(t_{2}\right)+m_{q_{L}}^{2}\left(t_{3}\right)\right)+\frac{1}{2}\left(m_{3}\right)\right)+\frac{1}{2}\left(m_{L^{2}}^{2}\left(t_{2}\right)+m_{L^{2}}^{2}\left(t_{3}\right)\right)- \\
\left.\frac{1}{4}\left(t_{1}\right)+m_{L^{1}}^{2}\left(t_{2}\right)\right)-\frac{1}{2} \mid
\end{array}
\end{array}
$$




$$
\begin{array}{r}
\mid \frac{1}{2}\left(m_{U_{R}}^{2}\left(t_{2}\right)+m_{U_{R}}^{2}\left(t_{3}\right)\right)+\frac{1}{2}\left(m_{Q_{L}}^{2}\left(t_{2}\right)+m_{Q_{L}}^{2}\left(t_{3}\right)\right)- \\
\frac{1}{4}\left(m_{L^{3}}^{2}\left(t_{1}\right)+m_{L^{3}}^{2}\left(t_{2}\right)\right)-\frac{1}{2} \mid= \\
\mid \frac{1}{2}\left(m_{L^{2}}^{2}\left(t_{2}\right)+m_{L^{2}}^{2}\left(t_{3}\right)\right)+\frac{1}{2}\left(m_{E_{R}^{2}}^{2}\left(t_{2}\right)+m_{E_{R}^{2}}^{2}\left(t_{3}\right)\right)+ \\
\frac{1}{4}\left(m_{L^{2}}^{2}\left(t_{1}\right)+m_{L^{2}}^{2}\left(t_{2}\right)\right)-\frac{1}{2} \mid= \\
\mid \frac{1}{2}\left(m_{E_{R}^{1}}^{2}\left(t_{2}\right)+m_{E_{R}^{1}}^{2}\left(t_{3}\right)\right)+\frac{1}{2}\left(m_{L^{3}}^{2}\left(t_{2}\right)+m_{L^{3}}^{2}\left(t_{3}\right)\right)+ \\
\frac{1}{4}\left(m_{L^{2}}^{2}\left(t_{1}\right)+m_{L^{2}}^{2}\left(t_{2}\right)\right)-\frac{1}{2} \mid
\end{array}
$$




\section{References}

[1] For reviews on string phenomenology see (and references there in):

L. E. Ibáñez, hep-ph/9911499;

F. Quevedo, hep-ph/9707434; hep-th/9603074;

I. Antoniadis, hep-th/0102202, E. Dudas, hep-ph/0006190;

K. Dienes, hep-ph/0004129:hep-th/9602045

[2] R. Blumenhagen, L. Görlich, B. Körs and D. Lüst, "Noncommutative compactifications of type I strings on tori with magnetic background flux", JHEP 0010 (2000) 006, hep-th/0007024: "Magnetic Flux in Toroidal Type I Compactification", Fortsch. Phys. 49 (2001) 591, hep-th/0010198

[3] R. Blumenhagen, B. Körs and D. Lüst, "Type I Strings with F and B-flux", JHEP 0102 (2001) 030, hep-th/0012156.

[4] J. Ellis, S. Kelley, D. V. Nanopoulos, Phys. Lett. B249 (1990) 441; U. Amaldi, W. De Boer and H. Fürstenau, Phys. Lett. B260 (1991) 131; P. Langacker and M. Luo, Phys. Rev. D44 (1991) 817

[5] L. Dixon, V. Kaplunovsky and J. Louis, Nucl Phys. B355 (1991) 649;

C. Kokorelis, "String Loop Threshold Corrections for N=1 Generalized Coxeter Orbifolds", Nucl.Phys. B579 (2000) 267-274, hep-th/0001217;

D. Bailin, A. Love, W.A. Sabra, S. Thomas, "String Loop threshold corrections for $Z_{N}$ Coxeter orbifolds", Mod. Phys. Lett. A9 (1994) 67-80, hep-th/9310008;

C. Kokorelis, "Gauge and Gravitational Couplings from Modular Orbits in Orbifold Compactifications", Phys. Lett. B477 (2000) 313, hep-th/0001062

G. L. Cardoso, D. Lüst, T. Mohaupt, "Threshold Corrections and Symmetry Enhancement in String Compactifications" Nucl.Phys. B450 (1995) 115, hep-th/9412209

[6] I. Antoniadis, N. Arkadi-Hamed, S. Dimopoulos, G. Dvali, Phys. Lett. B436 (1998) 257, hep-ph/9804398; I. Antoniadis, C. Bachas, Phys. Lett. B450 (1999) 83

[7] M. Berkooz, M. R. Douglas, R.G. Leigh, "Branes Intersecting at Angles", Nucl. Phys. B480 (1996) 265, hep-th/9606139

[8] M. Bianchi, G. Pradisi and A. Sagnotti, "Toroidal compactification and symmetry breaking in open string theories," Nucl. Phys. B376, 365 (1992); Z. Kakushadze, G. Shiu and S.-H. H. Tye, "Type IIB orientifolds with NS-NS antisymmetric tensor backgrounds," Phys. Rev. D58, 086001 (1998). hep-th/9803141; C. Angelantonj, Nucl. Phys. B566 (2000) 126, "Comments on Open-String Orbifolds with a Non-Vanishing $B_{a b}$ ", hepth/9908064 
[9] R. Blumenhagen, L. Görlish, and B. Körs, "Asymmetric Orbifolds, non-commutative geometry and type I string vacua", Nucl. Phys. B582 (2000) 44, hep-th/0003024

[10] C. Angelantonj and A. Sagnotti, "Type I vacua and brane transmutation", hepth/00010279;

C. Angelantonj, I. Antoniadis, E. Dudas and A. Sagnotti, "Type I strings on magnetized orbifolds and brane transmutation", Phys. Lett. B489 (2000) 223, hep-th/0007090

[11] L. E. Ibáñez, F. Marchesano and R. Rabadán, "Getting just the Standard Model at Intersecting Branes" JHEP, 0111 (2001) 002, hep-th/0105155;

[12] C. Kokorelis, "New Standard Model Vacua from Intersecting Branes", hep-th/0205147

[13] C. Kokorelis, "GUT Model Hierarchies from Intersecting Branes", hep-th/0203187

[14] R. Blumenhagen, L. Görlich, B. Körs, "Supersymmetric Orientifolds in 6D with DBranes at Angles", Nucl.Phys. B569 (2000) 209, hep-th/9908130; R. Blumenhagen, L. Görlich, B. Körs, "Supersymmetric 4D Orientifolds of Type IIA with D6-branes at Angles", JHEP 0001 (2000) 040,hep-th/9912204; R. Blumenhagen, B. Körs, D. Lüst and T. Ott, "The Standard Model from Stable Intersecting Brane World Orbifolds", Nucl. Phys. B616 (2001) 3, hep-th/0107138

[15] G. Aldazabal, S. Franco, L. E. Ibáñez, R. Rabadán and A. M. Uranga, "D=4 chiral string compactifications from intersecting branes", J. Math. Phys. 42 (2001) 3103-3126, hep-th/0011073; G. Aldazabal, S. Franco, L. E. Ibáñez, R. Rabadán and A. M. Uranga, "Intersecting brane worlds", JHEP 0102 (2001) 047, hep-ph/0011132.

[16] Stefan Forste, Gabriele Honecker, Ralph Schreyer, "Supersymmetric $Z_{N} \times Z_{M}$ Orientifolds in 4D with D-Branes at Angles", Nucl.Phys. B593 (2001) 127-154, hepth/0008250; Ion V. Vancea, "Note on Four Dp-Branes at Angles", JHEP 0104:020,2001, hep-th/0011251;

[17] H. Kataoka, M. Shimojo, “ $S U(3) \times S U(2) \times U(1)$ Chiral models from Intersecting D4-/D5-branes", hep-th/0112247; G. Honecher, "Non-supersymmetric Orientifolds with D-Branes at Angles", hep-th/0112174 G. Honecher, "Intersecting brane world models from D8-branes on $\left(T^{2} \times T^{4} / Z_{3}\right) / \Omega \mathcal{R}_{1}$ type IIA orientifolds", hep-th/0201037;

[18] D. Cremades, L. E. Ibáñez, F. Marchesano, 'SUSY Quivers, Intersecting Branes and the Modest Hierarchy Problem', hep-th/0201205; D. Cremades, L. E. Ibanez and F. Marchesano, "Standard Model at Intersecting D4/D5 Branes: Lowering the String Scale", hep-th/0205074; 
[19] D. Cremades, L. E. Ibanez and F. Marchesano, " Intersecting Brane Models of Particle Physics and the Higgs Mechanism", hep-th/0203160;

[20] L. F. Alday and G. Aldazabal, "In quest of "just" the Standard Model on D-branes at a singularity", JHEP 0205 (2002) 022, hep-th/0203129

[21] G Altazabal, L. E. Ibáñez, A. M Uranga, "Gauging Away the String CP problem", hep-ph/0205250

[22] R. Blumenhagen, V. Braun, B. Körs, D. Lüst " Orientifolds of K3 and Calabi-Yau Manifolds with Intersecting D-branes", hep-th/0206038

[23] M. Cvetic, G. Shiu A M. Uranga, "Chiral four dimensional $N=1$ supersymmetric IIA orientifolds from intersecting D6 branes", Nucl. Phys. B615 (2001) 3, hep-th/0107166; M. Cvetic, G. Shiu A M. Uranga, "Three family supersymmetric standard models from intersecting brane worlds", Phys. Rev. Lett. 87 (2001) 201801, hep-th/0107143;

M. Cvetic, G. Shiu A M. Uranga, "Chiral type II orientifold constructions as M theory on $G_{2}$ holonomy spaces", hep-th/0111179; M. Cvetic, P. Laugacker, G. Shiu, "Phenomenology of a Three family Standard-like String Model ", hep-ph/0205252; M. Cvetic, P. Langacker, G. Shiu, "A Three-Family Standard-like Orientifold Model: Yukawa Couplings and Hierarchy", hep-th/0206115

[24] R. Blumenhagen, B. Körs, D. Lüst and T. Ott, "The Standard Model from Stable Intersecting Brane World Orbifolds", Nucl. Phys. B616 (2001) 3, hep-th/0107138

[25] A. Sagnotti, "A Note on the Green - Schwarz Mechanism in Open - String Theories", Phys. Lett. B294 (1992) 196, hep-th/9210127

[26] L. E. Ibáñez, R. Rabadán and A. M. Uranga, "Anomalous U(1)'s in Type I and Type IIB $\mathrm{D}=4, \mathrm{~N}=1$ string vacua", Nucl.Phys. B542 (1999) 112-138

[27] A. Sen, JHEP 9808 (1998) 012, "Tachyon Condensation on the Brane Antibrane System" hep-th/9805170; "SO(32) Spinors of Type I and Other Solitons on Brane-Antibrane Pair", JHEP 809 (1998) 023, hep-th/9808141.

[28] P. Hernandez, K. Jansen, L. Lellouch, H. Wittig, "Scalar condensate and light quark masses from overlap fermions", Nucl. Phys. Proc. Suppl. 106 (2002) 766, hep-lat/0110199

[29] Heron of Alexandria, "Metrica", Book I, 50 A.D

[30] R. Blumenhagen, B. Körs, D. Lüst and T. Ott, "Moduli Stabilization for Intersecting Brane Worlds in type 0' String Theory", hep-th/0202124 
[31] J. Garcia-Bellido and R. Rabadan, "Complex Structure Moduli Stability in Toroidal Compactifications", JHEP 0205 (2002) 042, hep-th/0203247

[32] W. Fischer and L. Susskind, Phys. Lett. B171 (1986) 383; Phys. Lett. B173 (1986) 262

[33] E. Dudas and J. Mourad, "Brane solutions in strings with broken supersymmetry and dilaton tadpoles", Phys. Lett. B486 (2000) 172, hep-th/0004165; R. Blumenhagen, A. Font, "Dilaton tadpoles, warped geometries and large extra dimensions for nonsupersymmetric strings", Nucl. Phys. B 599 (2001) 241, hep-th/0011269. 